\section{Neuerwerbungen des LÖGD}

Iliffe S, Deppe HU. Health care in Europe: Competition or solidarity? Proceedings of the 9th Conference of the International Association for the Study of Health Policy (Europe), Thessaloniki, September 27th to October 1st, 1995. Frankfurt: VAS-Verlag für Akademische Schriften 1996. 149 S. ISBN 388864-999-4.

Peterender F, Speck O, Hrsg. Frühförderung in Europa. München: Reinhard 1996. 178 S. ISBN 3-497-01379-X.

Europäische Gesundheitssysteme und Pharmamärkte: Fakten und Trends. Eine aktuelle Dokumentation der Arzneimittel Zeitung. Neu-Isenburg: Ärzte Zeitung Verlagsgesellschaft 1996. 105 S. ISBN 3-928748-09-2.

Fachverband Sucht, Hrsg. Sucht und Erwerbsfähigkeit: wie erfüllen Rehabilitationseinrichtungen ihren Auftrag? Beiträge des 8 . Heidelberger Kongresses 1995. Geesthacht: Neuland Verlagsgesellschaft 1996. 368 S. ISBN 3-87581149-6.

Friedrich $\mathrm{H}$ et al. Krebs und seine psychischen und sozialen Folgen: Formen der Hilfestellung, Beratung und Therapie. Stuttgart: Thieme 1996.173 S. ISBN 3-13-103931-0.

Grossmann R, Hrsg. Gesundheitsförderung und Public Health: öffentliche Gesundheit durch Organisation entwickeln. Wien: Facultas Universitätsverlag 1996. 414 S. ISBN 3-85076-396-X.

Sonneck G, Hrsg. Krisenintervention und Suizidverhütung: ein Leitfaden für den Umgang mit Menschen in Krisen. Wien: Facultas Universitätsverlag 1995. 302 S. ISBN 3-85076-356-0.

The Club of Cologne; World Health Organization, Hrsg. Gesundheitsförderung und körperliche Aktivität: wissen-

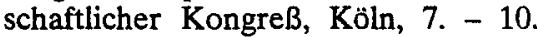
April 1994. Kongreßbericht. Köln: Verlag Sport und Buch Strauß 1996. 226 S. ISBN 3-89001-048-2.

Balz E. Gesundheitserziehung im Schulsport: Grundlagen und Möglich- keiten einer diätetischen Praxis. Schorndorf: Hofmann 1995. 232 S. ISBN 3-7780-8271-X.

Stegmüller K. Wettbewerb im Gesundheitswesen: Konzeptionen zur „dritten Reformstufe" der Gesetzlichen Krankenversicherung. Frankfurt: VAS Verlag 1996. 333 S. ISBN 3-88864-207-8.

Borsi GM, Schröck R. Pflegemanagement im Wandel: Perspektiven und Kontroversen. Berlin: Springer 1995. 348 S. ISBN 3-540-58642-3.

Brombie IK, Davies HTO. Research in health care: design, conduct and interpretation of health services research. Chichester: Wiley 1996. 288 S. ISBN 0471-96259-7.

Bauch J. Gesundheit als sozialer Code: von der Vergesellschaftung des Gesundheitswesens zur Medikalisierung der Gesellschaft. Weinheim: Juventa Verlag 1996. 192 S. ISBN 3-7799-1167-1.

Hamburger Projektgruppe Gesundheitsberichterstattung, Hrsg. Praxishandbuch Gesundheitsberichterstattung: ein Leitfaden für GesundheitsberichterstatterInnen und soiche, die es werden wollen. Düsseldorf: Akademie für Öffentliches Gesundheitswesen 1996. 272 S. ISBN 3-9804547-2-X.

Seiffge-Krenke I et al. Chronisch kranke Jugendliche und ihre Familien: Belastung, Bewältigung und psychosoziale Folgen. Stuttgart: Kohlhammer 1996. 313 S. ISBN 3-17-013885-5.

Krämer A, Stock C, Hrsg. HIV-Ausbreitung und Prävention: epidemiologische Trends und präventive Strategien. Weinheim: Juventa Verlag 1996. 206 S. ISBN 3-7799-1169-8.

Ley S, Stück B, Hrsg. Masern, Mumps, Röteln: Verbesserung der Durchimpfungsraten. Marburg: Kilian 1995.113 S. ISBN 3-9804445-5-4.

Webb GP. Nutrition: a health promotion approach. London: Arnold, 1995: 374 S. ISBN 0-340-61027-1.

Gabanyi M. Qualitätssicherung in der ambulanten Pflege. Augsburg: BASYS, 1995: 105 S. ISBN 3-930077-09-4. 